\title{
New Technique of Intracorporeal Anastomosis and Transvaginal Specimen Extraction for Laparoscopic Sigmoid Colectomy
}

\author{
Zheng Wang, Xing-Mao Zhang, Hai-Tao Zhou, Jian-Wei Liang, Zhi-Xiang Zhou*
}

\begin{abstract}
Background: Despite the growing acceptance of laparoscopic colon surgery, an abdominal incision is needed to remove the specimen and perform an anastomosis. Recently, natural orifice specimen extraction (NOSE) and intracorporeal anastomosis have been proposed to minimize abdominal wall trauma and improve the quality of laparoscopic colon resections Objective: To evaluate the feasibility and safety of a new approach combining intracorporeal delta-shaped anastomosis and transvaginal specimen extraction for totally laparoscopic sigmoid colectomy. Materials and Methods: Mobilization of bowel and dissection of lymph nodes were performed laparoscopically. After both proximal and distal incisal edges about $10.0 \mathrm{~cm}$ distance from sigmoid neoplasm were transected with an Endoscopic Linear Cutter-Straight, a small incision about $1.0 \mathrm{~cm}$ was created on the each colon wall of the contralateral side of the mesentery. Then anvils of an Endoscopic Linear Cutter-Straight were inserted into each colon through the small incisions, and incision and anastomosis between the walls of each colon were performed with a linear stapler. A V-shaped anastomosis was made on the wall and the remnant openings was reclosed with the Endoscopic Linear Cutter-Straight. The culdotomy was enlarged with laparoscopic ultrasound dissector. Transvaginal extraction of specimens was accomplished through a wound protector. Results: Surgery was performed for 11 patients with sigmoid cancer. No intraoperative complications or conversions occurred. The mean operating time was $132 \mathrm{~min}$. All the patients were treated laparoscopically without any postoperative complications. Conclusions: The procedures of intracorporeal delta-shaped anastomosis and transvaginal specimen extraction are safe and oncologically acceptable for selected colon cancer cases.
\end{abstract}

Keywords: Totally laparoscopic sigmoid colectomy - intracorporeal delta-shaped anastomosis - transvaginal extraction

Asian Pac J Cancer Prev, 15 (16), 6733-6736

\section{Introduction}

Colorectal carcinoma is the major cause of cancer related deaths in asia (Missaouia et al., 2010; Hwang, 2013 Li et al., 2013). In 1991, Jacobs et al (Jacobs et al., 1991) reported the initial experience of 20 cases of laparoscopic colectomy and proved its technical feasibility. After nearly 20 years of development, laparoscopic surgery for colon cancer has emerged as the standard procedure compared to open surgery with similar oncologic outcomes and superior perioperative results. Usually, an abdominal incision is required for anastomosis and specimen extraction for laparoscopic colon surgery.

Recently, natural orifice specimen extraction (NOSE) and intracorporeal anastomosis have been proposed to improve the quality of laparoscopic colon resections. This approach can eliminate a larger abdominal incision other than that for trocar placement (Franklin et al., 2000; Franklin et al., 2008). However, intracorporeal anastomosis is the major challenge in laparoscopic surgery.
Technique of delta-shaped anastomosis which was used for the gastric cancer surgery was reported firstly in 2002 (Kanaya et al., 2002), and there was no report on the feasibility and safety of totally laparoscopic resection with delta-shaped anastomosis for colon cancer surgery till now. In this report, we describe this simple and safe technique of intracorporeally delta-shaped anastomosis in sigmoid colectomy combined with transvaginal extraction of the specimen.

\section{Materials and Methods}

\section{Patients}

Totally laparoscopic sigmoid colectomy with intracorporeally delta-shaped anastomosis and transvaginal specimens extraction was indicated for patients who previously underwent vaginal delivery with clinical stage T1-T3 located in the sigmoid colon. We limited the indication to menopausal women only. Between January 2013 and January 2014, eleven patients with sigmoid 


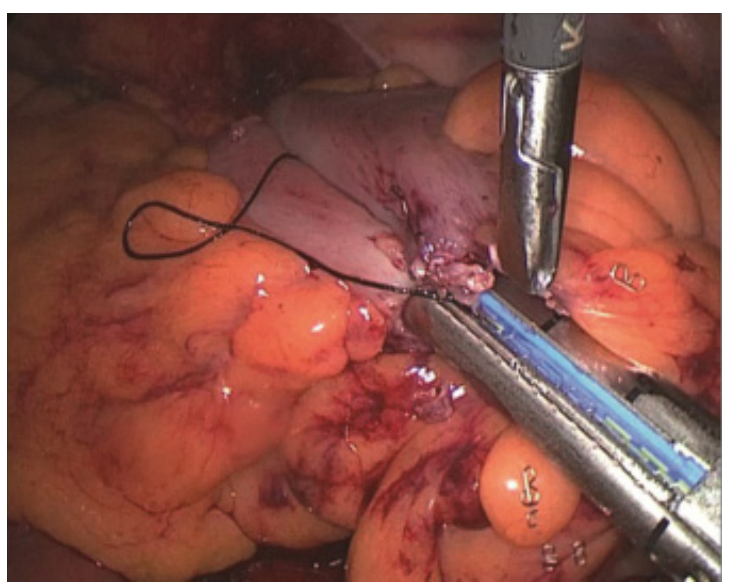

Figure 1. Incision and Anastomosis between the Walls of Each Colon Were Performed by the Endoscopic Linear Cutter-Straight

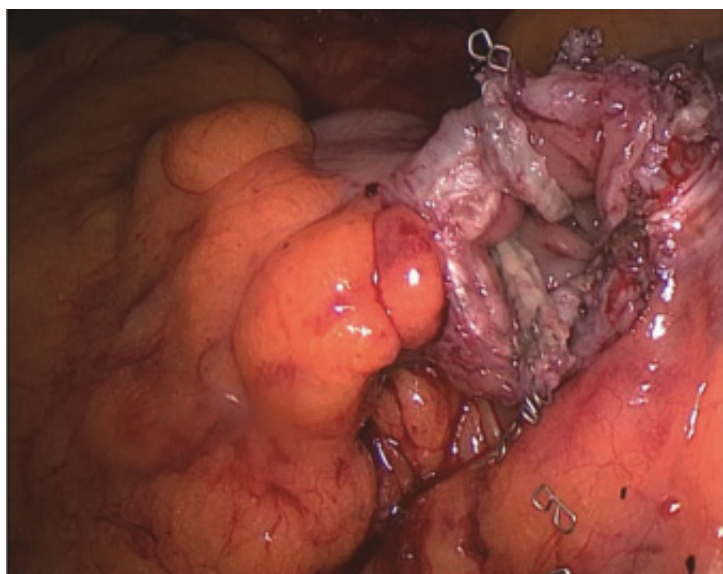

Figure 2. A V-Shaped Anastomosis was Made on the Wall

cancer underwent this procedure. All procedures were performed or supervised by surgeons experienced in both laparoscopic and colorectal surgery. Institutional review board approval was obtained before conducting this study. Patients gave their written informed consent to these procedure with intracorporeally delta-shaped anastomosis and transvaginal specimens extraction.

\section{Operative techniques}

The patients were placed in the modified lithotomy position, and the abdomen was then insufflated to 12 $\mathrm{mmHg}$. A four-port technique was used, and the following ports were placed: a 12-mm blunt-tip port beside the navel, 5-mm ports in the upper right and lower left abdominal quadrants, and a $12-\mathrm{mm}$ port in the right lower quadrant.

The small bowel was displaced from the pelvis with Trendelenburg and right tilt applied. The sigmoid colon was retracted towards the abdominal wall and the foot of the sigmoid mesocolon entered via a medial to lateral dissection. The inferior mesenteric artery and vein were defined and transected with laparoscopic linear staplers. The lateral colonic attachments were then freed along the line of Toldt to completely mobilize the sigmoid, distal part of the descending colon and the upper rectum. Splenic flexure mobilization was required in one case in which the lesion was located near the descending colon. The ureters,

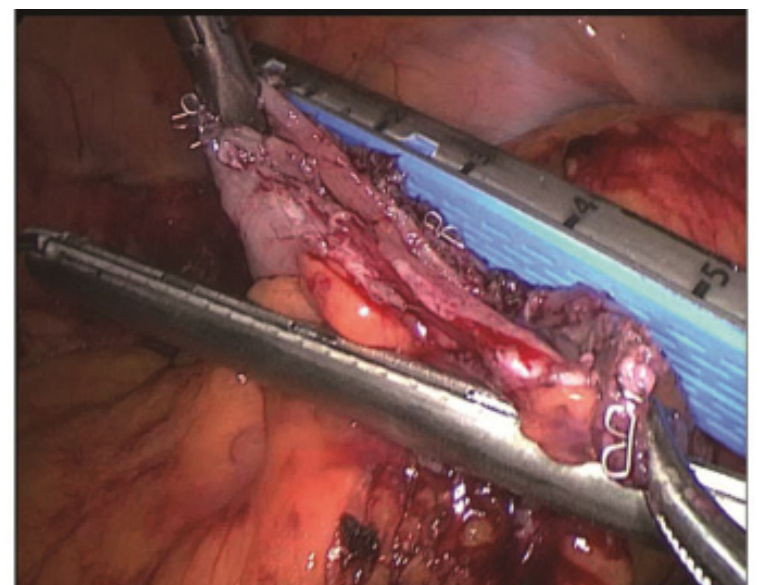

Figure 3. The Remanent Openings was Reclosed by an Endoscopic Linear Cutter-Straight

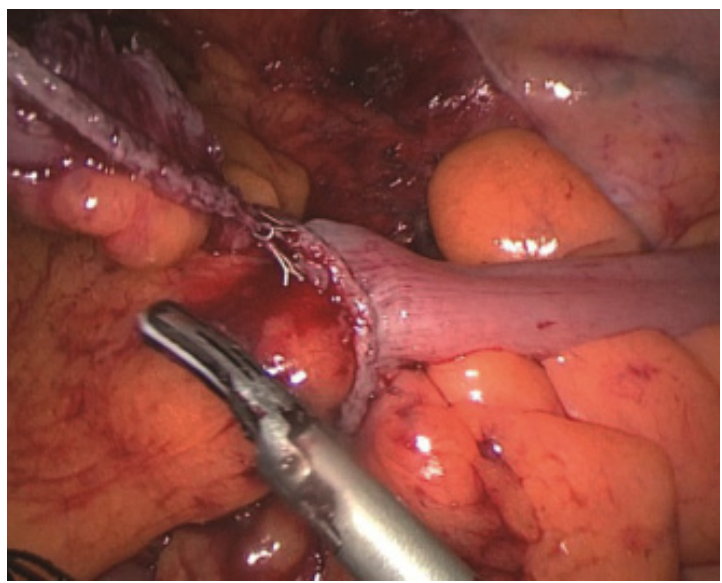

Figure 4. Laparoscopic View of the Completed Anastomosis

the hypogastric nerves, and the pelvic parasympathetic plexus were safeguarded. After both proximal and distal incisal edge about $10.0 \mathrm{~cm}$ distance from sigmoid neoplasm were transected by Endoscopic Linear CutterStraight, a small incision about $1.0 \mathrm{~cm}$ was created on the each colon wall of the contralateral side of mesentery. Then the upper and lower anvils of an Endoscopic Linear Cutter-Straight were inserted into each colon through the small incision respectively, and incision and anastomosis between the walls of each colon were performed by the linear stapler (Figure 1). A V-shaped anastomosis was made on the wall (Figure 2). The remanent openings was reclosed by an Endoscopic Linear Cutter-Straight (Figure $3,4)$. The culdotomy was enlarged with laparoscopic ultrasound dissector. Transvaginal extraction of specimens was accomplished through a wound protector (Figure 5). The culdotomy was closed laparoscopically with a running suture. After the pelvic cavity has been irrigated with 1 liters saline, a pelvic drain was inserted through the incision in the right lower quadrant.

\section{Results}

For eleven patients, totally laparoscopic sigmoid colectomy with intracorporeally delta-shaped anastomosis and transvaginal specimens extraction was attempted 
New Technique of Intracorporeal Anastomosis and Transvaginal Specimen Extraction for Laparoscopic Sigmoid Colectomy

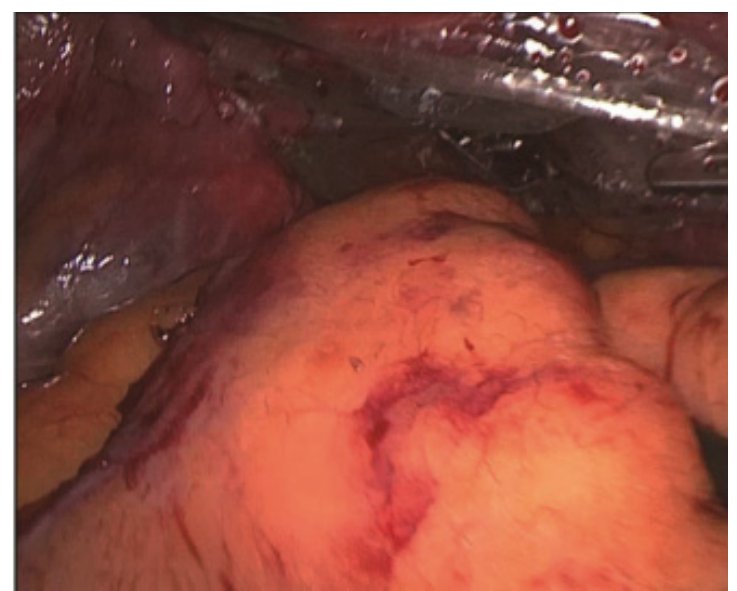

Figure 5. Transvaginal Extraction of Specimens was Accomplished Through A Wound Protector

Table 1. Patient Characteristics

\begin{tabular}{lllllllll}
\hline $\begin{array}{c}\text { Case Age } \\
\text { (years) }\end{array}$ & $\begin{array}{c}\text { BMI } \\
(\mathrm{kg} / \mathrm{m} 2)\end{array}$ & $\begin{array}{c}\text { Tumor } \\
\text { size } \\
(\mathrm{cm})\end{array}$ & $\begin{array}{c}\text { Stage } \\
\text { Operation } \\
\text { time } \\
(\mathrm{min})\end{array}$ & $\begin{array}{c}\text { Blood } \\
\text { loss } \\
(\mathrm{ml})\end{array}$ & $\begin{array}{c}\text { No. of lymph POHS } \\
\text { nodes } \\
\text { harvested }\end{array}$ & \\
\hline 1 & 54 & 21.5 & 2.5 & T2N0 & 125 & 20 & 18 & 8 \\
2 & 58 & 25 & 4 & T2N0 & 140 & 10 & 15 & 9 \\
3 & 60 & 30 & 3.4 & T2N1 & 135 & 12 & 17 & 7 \\
4 & 70 & 27.6 & 3.5 & T3N1 & 143 & 15 & 14 & 8 \\
5 & 46 & 28 & 3.6 & T2N1 & 130 & 25 & 15 & 8 \\
6 & 56 & 25.8 & 4 & T2N0 & 120 & 23 & 14 & 8 \\
7 & 52 & 25.1 & 2.5 & T2N1 & 123 & 15 & 12 & 7 \\
8 & 49 & 23.5 & 2.8 & T2NO & 113 & 25 & 15 & 7 \\
9 & 65 & 24.5 & 3 & T1N1 & 120 & 30 & 15 & 8 \\
10 & 70 & 25.5 & 3.5 & T2N1 & 125 & 24 & 16 & 9 \\
11 & 63 & 27.3 & 2.4 & T1N1 & 115 & 15 & 13 & 7 \\
\hline
\end{tabular}

and completed successfully (Table 1). The operations were completed laparoscopically without any serious perioperative complications. No intraoperative vaginal injuries were encountered. The average operation time was $126 \mathrm{~min}$ (range 113-143 $\mathrm{min}$ ), and the average blood loss was $19.4 \mathrm{ml}$ (range $10-30 \mathrm{ml}$ ). The median number of harvested lymph nodes was 15 (range 12-18). Clear liquids were resumed on postoperative day 2 , and a soft diet was allowed on postoperative day 3 .

No major complications (e.g., anastomotic leakage, stenosis, or abdominal abscess) occurred. The average postoperative hospital stay was 8 days (range, 7-9 days), and no mortality occurred in this series. The followup period ranged from 3 to 15 months. Seven patient underwent postoperative chemotherapy. There was no evidence of metastasis.

\section{Discussion}

Laparoscopic surgery has had a major impact on the practice of colon surgery. Laparoscopically assisted colon resection for cancer has clear advantages in terms of earlier return of bowel function, decreased postoperative pain, decreased length of stay and morbidity, when compared to open resection. However, laparoscopically assisted colectomy still involves externalization of the bowel for resection and anastomosis, using the open access for specimen extraction.

Intracorporeal anastomosis procedures can now be performed because of advances in equipment and surgical techniques. The aim of intracorporeal anastomosis is to reduce the extension of the abdominal incision and minimizing wound-related complications, such as wound infection and incisional hernia. However, end to end colorectal reconstruction after laparoscopic sigmoid colectomy is commonly performed extraabdominally because of the complexity of intracorporeal colorectal anastomotic procedure using a circular stapler (Bergamaschi et al., 1997). We have developed a new method of intracorporeally delta-shaped anastomosis using only endoscopic linear staplers. It is a simple, easy, and time-saving technique. Technique of delta-shaped anastomosis which was used for the gastric cancer surgery was reported firstly in 2002 and our report described the details of totally laparoscopic resection with delta-shaped anastomosis for colon cancer surgery. Our initial results reveal that our technique for colorectal anastomosis is a safe procedure.

To maximize the benefits of minimal access colonic procedures, the natural orifice specimen extraction (NOSE), which does not involve extraction minilaparotomy, has been developed. Some limitations have been associated with transanal specimen extraction procedure, and the procedure may not be possible in patients with bulky tumors, a thick mesentery, a narrow rectum, or anal stenosis. In addition, the risk of damage to the function of the anal sphincter has not been fully investigated. So the vagina has been established as one of the preferred routes for specimen extraction in NOSE because of its improved healing and elasticity (Franklin et al., 2008; McKenzie et al., 2010). Breda et al. (Breda et al., 1993) in 1993 first performed transvaginal NOSE for a patient with a small, nonfunctional tuberculous kidney. Wilson et al. (Wilson et al., 2007) in 2007 used the transvaginal route after right hemicolectomy for right colon cancer. Dozois EJ et al (Dozois et al., 2008) in 2008 described a total colectomy with transvaginal extraction for hereditary nonpolyposis colon cancer.

We attempted to perform the procedure of intracorporeally delta-shaped anastomosis combined with the technique of transvaginal specimens extraction for sigmoid colon cancer and obtained good results. There is little data on this new technique previously. The benefits of this new technique include: reduced tissue trauma; absent abdominal incision for specimen extraction; less wound related complications. All these advantages lead to less pain, earlier return of bowel function and shorter hospital stay. This technique is therefore considered to be a more feasible and safe procedure for patients with sigmoid colon cancer. Intraabdominal opening of the bowel may increase the risk of abdominal abscess and vaginal metastasis, we used a protective barrier to reduce the incidence of these problems, although there are no reported cases in the literature; and there are no reports as well of complications related to the culdotomy. However, further prospective investigations are necessary to establish its superiority over the conventional laparoscopically assisted approach and other NOSE approach.

In our opinion, the use of intracorporeally delta-shaped anastomosis and transvaginal specimen extraction may 
allow a sigmoid colectomy without complications in suitable patients. However, we believe that it should be performed by a experienced expert surgeon. In the future, this method would be an ideal choice for the suitable patients.

\section{References}

Bergamaschi R, Arnaud JP (1997). Intracorporeal colorectal anastomosis following laparoscopic left colon resection. Surgical Endoscopy, 11, 800-1.

Breda G, Silvestre P, Giunta A, et al (1993). Laparoscopic nephrectomy with vaginal delivery of the intact kidney. Eur urology, 24, 116-7.

Dozois EJ, Larson DW, Dowdy SC, et al (2008). Transvaginal colonic extraction following combined hysterectomy and laparoscopic total colectomy: a natural orifice approach. Tech Coloproctol, 12, 251-4.

Franklin Jr M, JA DE (2000). Laparoscopic left hemicolectomy with transanal extraction of the specimen. Atlas of laparoscopic surgery WB Saunders Co, Philadelphia:386-404.

Franklin ME, Kelley H, Kelley M, et al (2008). Transvaginal extraction of the specimen after total laparoscopic right hemicolectomy with intracorporeal anastomosis. Surg Laparosc Endosc Percutan Tech 18, 294-8.

Hwang H (2013). Colorectal cancer screening among Asian Americans. Asian Pac J Cancer Prev, 14, 4025-32.

Jacobs M, Verdeja JC, Goldstein HS (1991). Minimally invasive colon resection (laparoscopic colectomy). Surg Laparosc Endosc, 1, 144-50.

Kanaya S, Gomi T, Momoi H, et al (2002). Delta-shaped anastomosis in totally laparoscopic Billroth I gastrectomy: new technique of intraabdominal gastroduodenostomy. $\mathrm{J} \mathrm{Am}$ Coll Surg, 195, 284-7.

Li XP, Xie ZY, Fu YF, et al (2013). Colorectal cancer concealment predicts a poor survival: a retrospective study. Asian Pac J Cancer Prev, 14, 4157-60.

McKenzie S, Baek JH, Wakabayashi M, et al (2010). Totally laparoscopic right colectomy with transvaginal specimen extraction: the authors' initial institutional experience. Surg endosc, 24, 2048-52.

Missaouia N, Jaidaine L, Ben Abdelkader A, et al (2010). Clinicopathological patterns of colorectal cancer in Tunisia. Asian Pac J Cancer Prev, 11, 1719-22.

Wilson JI, Dogiparthi KK, Hebblethwaite N, et al (2007). Laparoscopic right hemicolectomy with posterior colpotomy for transvaginal specimen retrieval. Colorectal Dis, 9, 662 . 